\title{
Phase Diagram of the Attractive Hubbard Model with Inhomogeneous Interactions
}

\author{
Vijay B. Shenoy \\ Centre for Condensed Matter Theory, Department of Physics, \\ Indian Institute of Science, Bangalore 560 012, India
}

(Dated: November 10, 2018)

\begin{abstract}
The phase diagram of the attractive Hubbard model with spatially inhomogeneous interactions is obtained using a single site dynamical mean field theory like approach. The model is characterized by three parameters: the interaction strength, the active fraction (fraction of sites with the attractive interaction), and electron filling. The calculations indicate that in a parameter regime with intermediate values of interaction strength (compared to the bare bandwidth of the electrons), and intermediate values of the active fraction, "non-BCS" superconductivity is obtained. The results of this work are likely to be relevant to many systems with spatially inhomogeneous superconductivity such as strongly correlated oxides, systems with negative $U$ centers, and, in future, cold atom optical lattices.

PACS numbers: 74.81.-g, 71.10.Fd, 74.20.-z, 74.25.Dw
\end{abstract}

\section{INTRODUCTION}

There is a growing body of experimental evidence that strongly correlated oxides such as cuprates,$\frac{1,2,3,4,5,6,7,8,9}{,}$ manganites $10,11,12,13,14,15$ etc., are electronically inhomogeneous. The term "electronically inhomogeneous" is used to describe states with spatially inhomogeneous electronic orders, i.e., of orders of charge, spin, superconducting gap etc. There are suggestions that such inhomogeneous electronic states are one of the characteristic features intrinsic to strongly correlated materials 16 arising out of their "electronic softness" $\underline{17}$ A clear understanding of this phenomenon could, for example, suggest possibilities of controlling the nature and size of the electronic inhomogeneities, and can lead to, inter alia, possible device applications of these materials. Efforts directed towards uncovering the physics of the origin and nature of electronically inhomogeneous electronic states, therefore, have emerged as a very active research area.

Of particular interest to this work is electronically inhomogeneous superconducting state which is found in many systems of current interest. High temperature superconducting cuprates are prominent examples of systems showing inhomogeneous superconductivity. The past five or so years have witnessed fascinating experimental work based on scanning probes that have revealed a wealth of information regarding the nature of the inhomogeneous superconducting state in

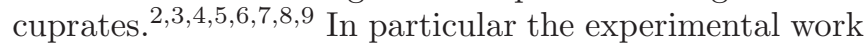
reported in references [8,9] has clearly demonstrated a distribution of gaps, and even regions with gaps above the superconducting transition temperatures. There could be several physical origins to this phonemenon, such as one body disorder due to the dopant ions, inhomogeneous pairing interactions etc. Superconductivity arising out of inhomogeneous pairing interactions are also found in many other systems. Anderson 18 suggested the possibility of negative- $U$ centres in semiconductors. There are reports of existence of superconductivity in silicon based nanostructures with negative- $U$ centres ${ }^{19}$ Negative- $U$ models have been used to describe

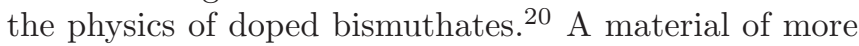
recent interest, $\mathrm{Tl}$-doped $\mathrm{PbTe}$, is believed to have a distribution of negative- $U$ centers ${ }^{21.22}$

As indicated briefly above, there are two factors that lead to an inhomogeneous superconducting state. The first one is one body disorder; when one body disorder is large, it tends to localize electrons, and in this sense "competes" with superconductivity $\underline{23}$ Effect of one body disorder on superconductivity has been extensively studied using models and methods of different sophistication $24,25,26$ The second factor that contributes to inhomogeneous superconductivity arises in situations where the pairing interaction (such as negative- $U$ centers) responsible for superconductivity is itself spatially inhomogeneous. Systems with a distribution of negative$U$ centers are known to give rise to the "charge Kondo effect" 27 ; the material Tl-doped PbTe is believed to be one such $\stackrel{28}{ }$ Models with inhomogeneous pairing interactions have been investigated before ${ }^{29}, 30,31,32,33,34,35,36$ The model usually studied is the attractive Hubbard model with inhomogeneous interactions (AHII) is described by the Hamiltonian:

$H=-t \sum_{<i j>\sigma}\left(c_{i \sigma}^{\dagger} c_{j \sigma}+\right.$ h.c. $)-\sum_{i} U_{i} n_{i \uparrow} n_{i \downarrow}-\mu \sum_{i \sigma} n_{i \sigma}(1)$

where $i, j$ are site indices of a lattice, $t$ is the hopping amplitude, $\sigma$ is the spin index, $c_{i \sigma}^{\dagger}$ is the electron operator which creates an electron of spin $\sigma$ at site $i, n_{i \sigma}=c_{i \sigma}^{\dagger} c_{i \sigma}$ is the number operator at site $i$ of spin $\sigma, \mu$ is the chemical potential. The interaction $U_{i} \geq 0$ is site dependent; a fraction $p$ (here called the active fraction) of sites have $U_{i}=U>0$, while $U_{i}=0$ for the other fraction $(1-p)$ of sites. These sites with $U_{i} \neq 0$ can be arranged periodically or randomly. It is known that for a given $U$, there is a critical value $p_{c}$ of $p$ below which superconductivity is killed $29,30,31$

More recently the above model (11), motivated by the electronic inhomogeneities in correlated materials, has been subjected to extensive numerical simulations $\underline{\underline{34,35,36}}$ 


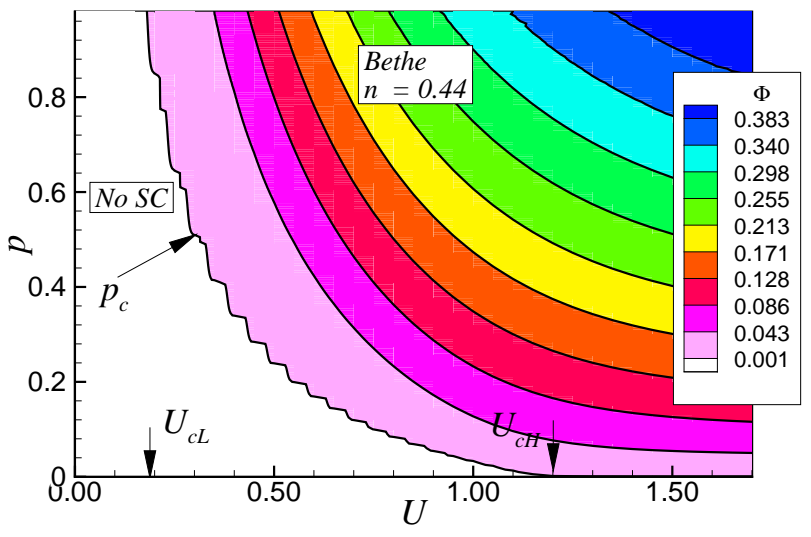

(a)

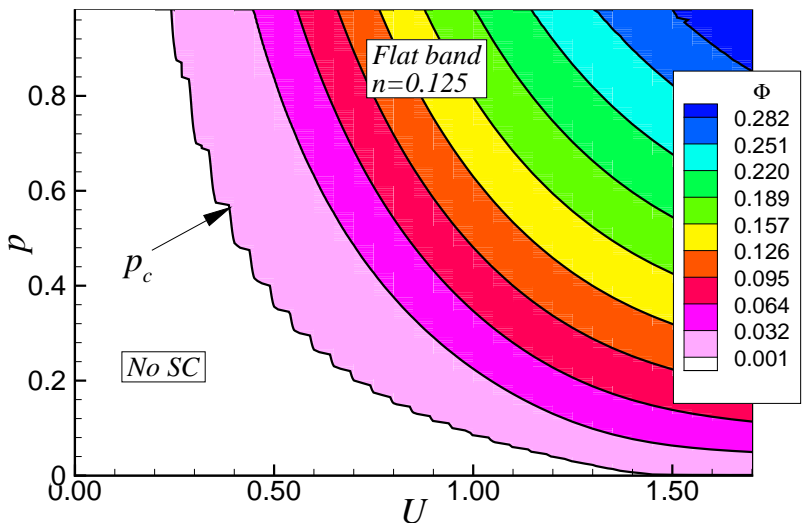

(b)

FIG. 1: (color online) $T=0$ phase diagram in the $p-U$ plane. Plots show contours of constant pairing $\Phi$. The pairing amplitude vanishes on the "left" side of the curve marked as $p_{c}$. (a) Bethe lattice with filling $n=0.44$ (b) Flat band with filling $n=0.125$.

A Bogoliubov-de Gennes mean-field (BdGMF) approach is used to obtain the ground state and finite temperature properties; these calculations involve averaging over several different $U$-disorder realizations. Quantum Monte Carlo calculations ${ }^{37}$ have also been performed on a twodimensional square lattice. Such calculations are numerically intensive, and attention has been focussed on particular values of electron fillings and interaction parameters $U$, and the active fraction $p$. It is useful to have a "phase diagram" of the AHII, particularly to compare and contrast different experimental systems, and to obtain regions in the parameter space where interesting physics may be expected. Calculation of the phase diagram within the BdGMF approach can be quite time consuming; it is therefore desirable to generate the phase diagram by means of a simple approach to understand its overall structure.

Motivated by the above discussion, the phase diagram of the AHII model is obtained in this paper using a dynamical mean field theory like approach. $\stackrel{38}{E}$ Effects of in-

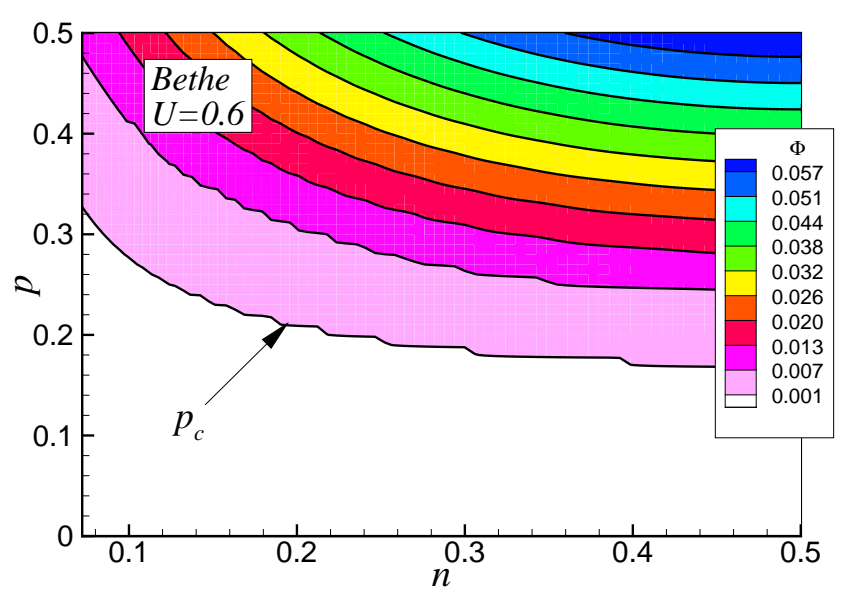

(a)

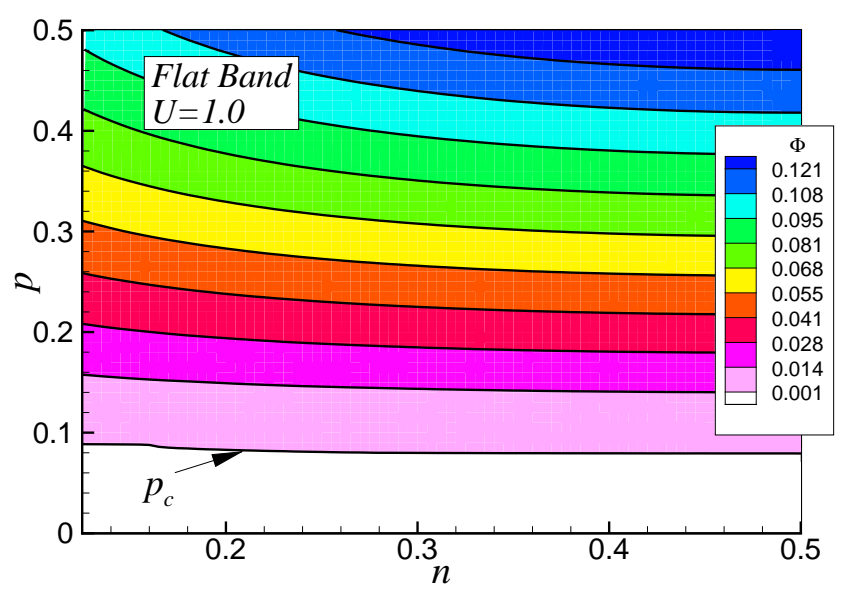

(b)

FIG. 2: (color online) $T=0$ phase diagram in the $p-\mu$ plane. Plots show contours of constant pairing $\Phi$. The pairing amplitude vanishes below the curve marked as $p_{c}$. (a) Bethe lattice with $U=0.6$ (b) Flat band with $U=1.0$.

teraction strength, active fraction and electron filling are investigated systematically. It is found that there are regions of the phase diagram where "unconventional" superconductivity is supported.

The paper is organized as follows. In the next section (Section II), we discuss the single site formulation of the problem. Results of the calculations are presented in Section [II] In the final section (Section IV) the results are discussed, and aspects of the phase diagram not captured by the present treatment, including a more speculative phase diagram, are discussed.

\section{FORMULATION}

The AHII Hamiltonian given in (1) is treated here within a dynamical mean field theory like approach. A dynamical mean field theory treatment of Hubbard like Hamiltonians with one body disorder is treated by 
Janiš and Vollhardt ${ }^{39}$; the present work deals with the case where the interaction term is disordered. A single site, that hybridizes with an electron bath ${ }^{38}$ described by a bath Green's function $\mathcal{G}$ (this is a matrix in the present formulation, see below), represents a typical site of the lattice. The imaginary time action for this site is written as

$$
\begin{aligned}
S\left[\Psi^{\star}, \Psi, V\right]= & -\int_{0}^{\beta} \int_{0}^{\beta} \mathrm{d} \tau \mathrm{d} \tau^{\prime} \Psi^{\star}(\tau) \mathcal{G}^{-1}\left(\tau-\tau^{\prime}\right) \Psi\left(\tau^{\prime}\right) \\
& -\int_{0}^{\beta} \mathrm{d} \tau V \psi_{\uparrow}^{\star}(\tau) \psi_{\downarrow}^{\star}(\tau) \psi_{\downarrow}(\tau) \psi_{\uparrow}(\tau)
\end{aligned}
$$

where $\beta=1 / T$ ( $T$ is the temperature), $\psi_{\sigma}$ are the Grassmann variables of the site electrons,

$$
\Psi=\left(\begin{array}{c}
\psi_{\uparrow} \\
\psi_{\downarrow}^{\star}
\end{array}\right), \Psi^{\star}=\left(\begin{array}{cc}
\psi_{\uparrow}^{\star} & \psi_{\downarrow}
\end{array}\right)
$$

are the Nambu matrices, $\mathcal{G}$ is the matrix Green's function (which incorporates the chemical potential $\mu$ ), $V$ is the attractive interaction. The Grassmann variables satisfy the Fermionic condition $\psi_{\sigma}(\beta)=-\psi_{\sigma}(0)$. The interaction $V$ is a random variable which here is distributed according to the probability distribution

$$
P(V)=(1-p) \delta(V)+p \delta(V-U)
$$

where $p$ is the active fraction, $\delta(\cdot)$ is the Dirac delta function, and $U$ is the attractive interaction strength at the active sites. The disorder averaged partition function can now be written as a disorder averaged path integral

$$
Z=\int \mathrm{d} V P(V) \int \mathcal{D}\left[\psi_{\sigma}^{\star}, \psi_{\sigma}\right] e^{-S\left[\Psi^{\star}, \Psi, V\right]}
$$

The site Green's function (expressed in terms of Matsubara frequencies $\left.i \omega_{n}\right)$ is obtained as

$$
\mathbb{G}\left(i \omega_{n}\right)=(1-p) \mathcal{G}\left(i \omega_{n}\right)+p\left(\mathcal{G}^{-1}\left(i \omega_{n}\right)-\Sigma_{U}\left(i \omega_{n}\right)\right)^{-1}(5)
$$

where $\Sigma_{U}$ is the self energy obtained from the solution of the quantum impurity problem with the bath Green's function $\mathcal{G}$ and an attractive Hubbard interaction $U$ at the site. The Green's function (5) represents the lattice Green's function as seen from the single site formulation. The site self energy is now given by

$$
\boldsymbol{\Sigma}\left(i \omega_{n}\right)=\mathcal{G}^{-1}\left(i \omega_{n}\right)-\mathbb{G}^{-1}\left(i \omega_{n}\right)
$$

Using the dynamical mean field theory ansatz ${ }^{38}$ that the the self energy is "momentum (energy) independent", we obtain the lattice Green's function $\mathscr{G}$ as

$$
\mathscr{G}\left(i \omega_{n}\right)=\int \mathrm{d} \varepsilon g(\varepsilon)\left(i \omega_{n} \mathbf{1}-\xi \boldsymbol{\tau}_{z}-\boldsymbol{\Sigma}\left(i \omega_{n}\right)\right)^{-1}
$$

where $\mathbf{1}$ is a $2 \times 2$ unit matrix, $\boldsymbol{\tau}_{z}$ is the Pauli $z$-matrix, $\xi=\varepsilon-\mu$, and $g(\varepsilon)$ is the bare density of states of the lattice. The dynamical mean field theory self consistency condition is now obtained by insisting that

$$
\mathcal{G}^{-1}\left(i \omega_{n}\right)=\mathscr{G}^{-1}\left(i \omega_{n}\right)+\boldsymbol{\Sigma}\left(i \omega_{n}\right) .
$$

This condition is obtained by demanding that the site Green's function as calculated from the quantum impurity formulation (5) is same as the lattice Green's function calculated via (7).

The following paragraphs contain a description of the approximate solution, based on the saddle point method, of the quantum impurity problem that is used in this work. A Hubbard-Stratanovich field $\Delta(\tau)$ is introduced to decouple the interaction term in the particle-particle channel. The partition function becomes

$$
Z=\int \mathrm{d} V P(V) \int \mathcal{D}\left[\Delta^{*}, \Delta\right] e^{-\int_{0}^{\beta} \mathrm{d} \tau|\Delta(\tau)|^{2}} \int \mathcal{D}\left[\psi_{\sigma}^{\star}, \psi_{\sigma}\right] e^{-S\left[\Psi^{\star}, \Psi, V, \Delta\right]}
$$

where

$$
S\left[\Psi^{\star}, \Psi, V, \Delta\right]=-\int_{0}^{\beta} \int_{0}^{\beta} \mathrm{d} \tau \mathrm{d} \tau^{\prime} \Psi^{\star}(\tau)\left(\mathcal{G}^{-1}\left(\tau-\tau^{\prime}\right)+\sqrt{V} \boldsymbol{\Delta}\left(\tau-\tau^{\prime}\right)\right) \Psi\left(\tau^{\prime}\right)
$$

with $\boldsymbol{\Delta}$ defined as

$$
\Delta\left(\tau-\tau^{\prime}\right)=\left(\begin{array}{cc}
0 & \Delta(\tau) \\
\Delta^{*}(\tau) & 0
\end{array}\right) \delta\left(\tau-\tau^{\prime}\right)
$$

The Fermionic path integral is easily evaluated, and the partition function becomes

$$
\int \mathrm{d} V P(V) \int \mathcal{D}\left[\Delta^{*}, \Delta\right] e^{-\left(\int_{0}^{\beta} \mathrm{d} \tau|\Delta(\tau)|^{2}-\ln \operatorname{det}\left[-\left(\mathcal{G}^{-1}+\sqrt{V} \boldsymbol{\Delta}\right)\right]\right)} .
$$


The averaging over the probability distribution can be performed exactly to obtain

$$
Z=\int \mathcal{D}\left[\Delta^{*}, \Delta\right] e^{-S_{\Delta}}
$$

where

$$
S_{\Delta}=\int_{0}^{\beta} \mathrm{d} \tau|\Delta(\tau)|^{2}-\ln \left[(1-p) \operatorname{det}\left(-\mathcal{G}^{-1}\right)+p \operatorname{det}\left(-\left(\mathcal{G}^{-1}+\sqrt{U} \boldsymbol{\Delta}\right)\right)\right] .
$$

The partition function is now evaluated by introduction of the saddle point approximation which amounts to treating $\Delta$ as independent of the imaginary time $\underline{\underline{40}}$; in the present context this approximation is equivalent to the BCS meanfield decoupling of the interaction term. With the assumption that $\Delta$ is real (equivalent to picking a particular phase of the resulting superconductor), the value of $\Delta$ is obtained by minimizing $S_{\Delta}$ leading to the equation

$$
\Delta=\frac{p e^{\ln \operatorname{det}\left[-\left(\mathcal{G}^{-1}+\sqrt{U} \boldsymbol{\Delta}\right)\right]}}{(1-p) e^{\ln \operatorname{det}\left[-\mathcal{G}^{-1}\right]}+p e^{\ln \operatorname{det}\left[-\left(\mathcal{G}^{-1}+\sqrt{U} \boldsymbol{\Delta}\right)\right]}}\left[\frac{\sqrt{U}}{2} \frac{1}{\beta} \sum_{i \omega_{n}}\left(\mathcal{G}_{12}^{\Delta}\left(i \omega_{n}\right)+\mathcal{G}_{21}^{\Delta}\left(i \omega_{n}\right)\right)\right]
$$

where $\left.\mathcal{G}^{\Delta}=\left(\mathcal{G}^{-1}+\sqrt{U} \boldsymbol{\Delta}\right)^{-1}\right)$.

Within this approximation the self energy $\Sigma_{U}$ in (5) is equal to $\sqrt{U} \Delta$, where the $\Delta$ obtained from the solution of (15) is used in (11).

In present formulation within a dynamical mean field theory framework, the saddle point approximation is the simplest possible "impurity solver". The formulation based on the Hubbard-Stratanovich fields is amenable to more sophisticated, and obviously more computationally intensive, treatments such as the Hrisch-Fye quantum monte carlo method. $\stackrel{41}{*}$ The saddle point approximation for the impurity solver is similar to the coherent potential approximation $\underline{42}$

In the framework developed here, $\sqrt{U} \Delta$ has the natural interpretation of the disorder averaged pairing gap. It should also be noted that there is a possibility of introducing a second Hubbard-Stratanovich field in the particle-hole channel, which in effect is equivalent to introducing an additional Hartree potential in the saddle point approximation. This extra Hartree potential can now be absorbed into the definition of the chemical potential $\mu$.

For a given value of $U$ and $p$, the value of $\Delta$ is calculated as follows. A typical calculation starts with an assumed value of $\Delta$, and a new value of $\Delta$ is calculated using (15). The site Green's function (5) and the self energy (6) are calculated using the new value of $\Delta$. The site self energy is used in (7) to obtain the lattice Green's function, and a new bath Green's function is generated using (8). This process is carried out until the values of $\Delta$, self energy $\boldsymbol{\Sigma}$ are within a specified tolerance of each other in two successive iterations. All self-consistency calculations are done at fixed chemical potential $\mu$ and the number of electrons $n$ is obtained after convergence is obtained. The chemical potential is then adjusted to that the number electrons is obtained to be the desired value. The calculations reported here are performed by evaluating all Matsubara sums as integrals along the real frequency axis, and the self consistency condition also enforced on the real frequency axis.

\section{RESULTS}

In the single site formulation presented in the last section, the information regarding the lattice enters the formulation only via the density of states $g(\varepsilon)$. Since the goal of this paper is to understand the generic features of the phase diagram of the AHII model, densities of states with simple analytical forms that capture some features of the real lattice systems are adopted. Two cases are considered. The semicircular density of states corresponding to a Bethé lattice $\frac{43}{3}$ with

$$
g(\varepsilon)=\frac{2}{\pi} \sqrt{1-\varepsilon^{2}}, \quad-1 \leq \varepsilon \leq 1
$$

and the flat band density of states

$$
g(\varepsilon)=\frac{1}{2}, \quad-1 \leq \varepsilon \leq 1 .
$$

Energy is measured in the units of half bandwidth of the systems, and hence the condition $-1 \leq \varepsilon \leq 1$ in both cases. Other parameter values of quantities such as $U, \mu, T$ are all henceforth dimensionless ratios of these quantities and the half bandwidth.

Superconductivity is monitored by computing the pairing amplitude $\Phi$ :

$$
\Phi=\left\langle\psi_{\uparrow}^{\star} \psi_{\downarrow}^{\star}\right\rangle=-\frac{1}{\pi} \int_{-\infty}^{\infty} \mathrm{d} \omega \Im \mathbb{G}_{12}(\omega)
$$

Vanishing of $\Phi$ implies absence of pairing and superconductivity. Clearly, existence of a nonzero value of $\Phi$ automatically does not imply global superconductivity. This point is discussed in more detail later in the paper when 


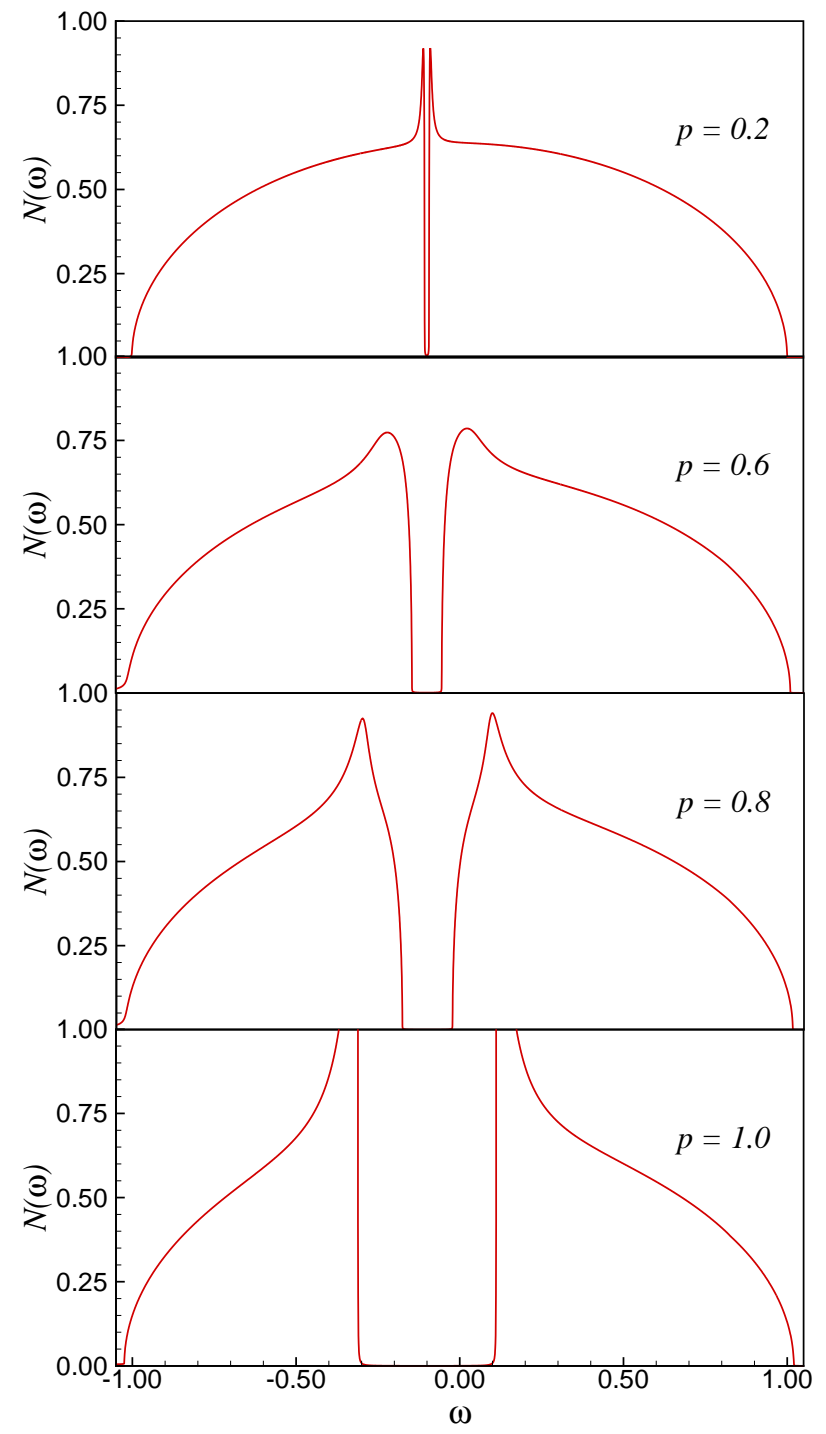

FIG. 3: (color online) Spectral function for the Bethe lattice with $U=0.8, n=0.44$. The four panels show the spectral function for increasing values of the active fraction $p$.

the Bose-Einstein condensation like phenomenon in such systems is discussed.

Results at zero temperature are presented first followed by results at $T>0$.

\section{A. Results: $T=0$}

For a given density of states, the phase diagram is determined by three parameters: the interaction strength $U$, the active fraction $p$ and the filling $n$. Fig. 1 shows the phase diagram of the system in the $p-U$ plane for different fillings. For a given filling $n$ we see that there is a range of interaction strength $U$ for which there is a critical value of the active fraction $p_{c}$ that is required to produce a nonzero pairing amplitude $\Phi$. In the case of the Bethe lattice the value of $p_{c}$ decreases with increasing filling $n$, while for the flat band case, $p_{c}$ is essentially insensitive to filling. This result is a reflection of the fact that $p_{c}$ is affected by the bare density of states (at the chemical potential); the bare density of states increases with increase in filling (up to $n \leq 0.5$ ) for the Bethe lattice, and hence the decrease of $p_{c}$. The critical active fraction $p_{c}$ is insensitive to filling in the flat band case since the density of states is constant. This is more clearly illustrated in Fig. 2 which shows the phase diagram in the $p-n$ plane for two values of $U$. It is evident that $p_{c}$ increases with decreasing $n$, and this increase is related to the decrease in the bare density of states (see Fig. 2(a)). Interestingly, the sensitivity of $p_{c}$ on $n$ decreases with increasing interaction strength $U$. The present calculation reveals an interesting new result. For values of $U$ larger than a critical value $U_{c H}$ (this value depends on the filling, i.e., bare density of state at the chemical potential), the critical value of the active fraction $p_{c}$ becomes vanishingly small (see Fig. 1). Thus if $U>U_{c H}$, even a small concentration of impurities can produce a non-zero pairing amplitude. In the same vein, there is another critical value of the interaction $U_{c L}$. If the interaction strength is below $U_{c L}$ (which, again, depends on the bare density of states at the chemical potential) even a small dilution of the active fraction from unity kills the pairing amplitude!

The spectral function,

$$
N(\omega)=-\frac{1}{\pi} \Im \mathbb{G}_{11}(\omega),
$$

also provides interesting information regarding the nature of the electronic state. Fig. 3 shows spectral functions for the Bethe lattice with $U=0.8, n=0.44$ (results for the case of the flat band qualitatively similar), for various values of the active fraction $p$. The spectral function at $p=1$ has a gap with a characteristic BCS (Bardeen-Cooper-Schrieffer) singularity $\underline{\underline{4}}$ in the spectral function near the gap edges. On the other hand for $p<1$ it is seen that the singularity is "smeared out", by appearance of "mid-gap states". The calculation suggests a possible spatial distribution of gaps in the system with different regions of the lattice developing different gaps. It is, of course, not possible within the present framework to study the gap distribution, but further detailed simulations could throw more light on the nature of the inhomogeneous state.

\section{B. Results at $T \neq 0$}

Fig. 4 shows the phase diagram of the AHII model in the $p-T$ plane. Several interesting features can be seen. For all active fractions with a non-vanishing pairing amplitude at zero temperature, there is a temperature $T_{c}$ at which the pairing amplitude (and $\Delta$ ) vanishes. There are three regimes of active fraction that give rise to very different finite temperature phenomenon. For active fractions just above the critical value (marked $p_{c}$ in 


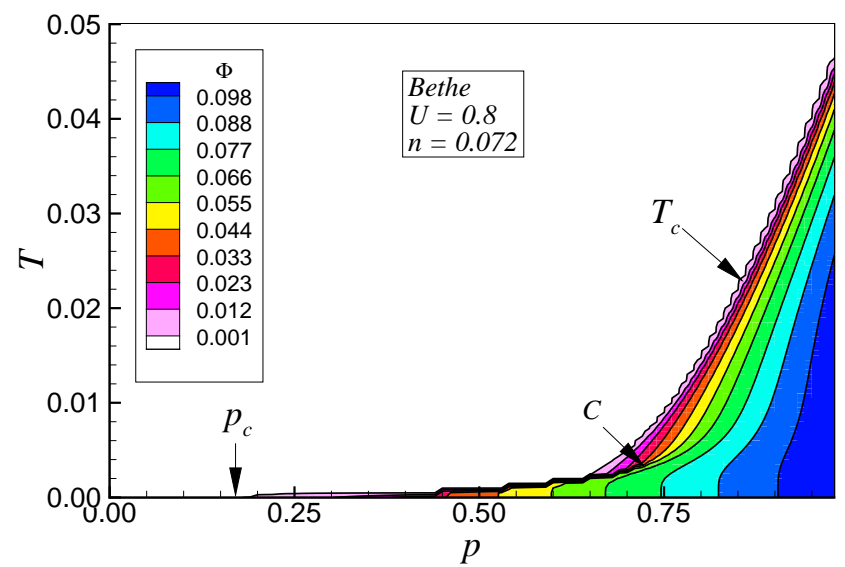

(a)

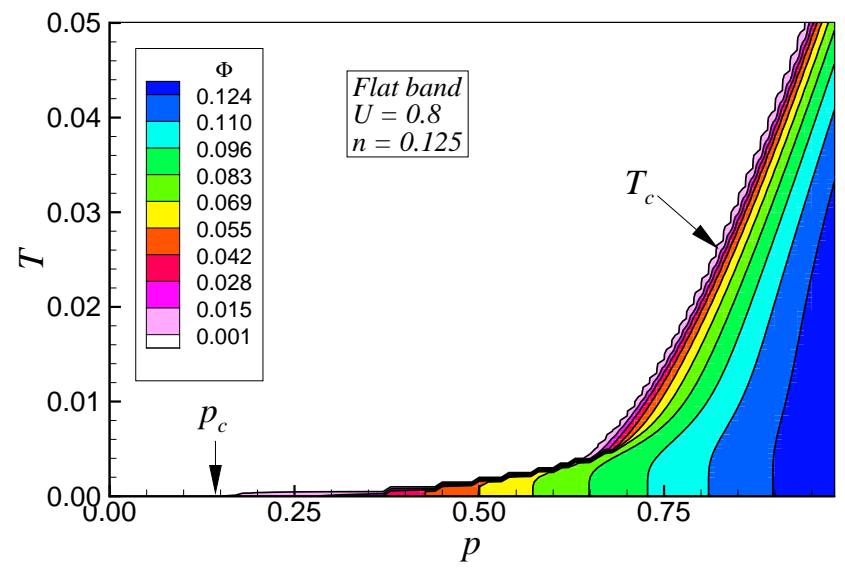

(c)

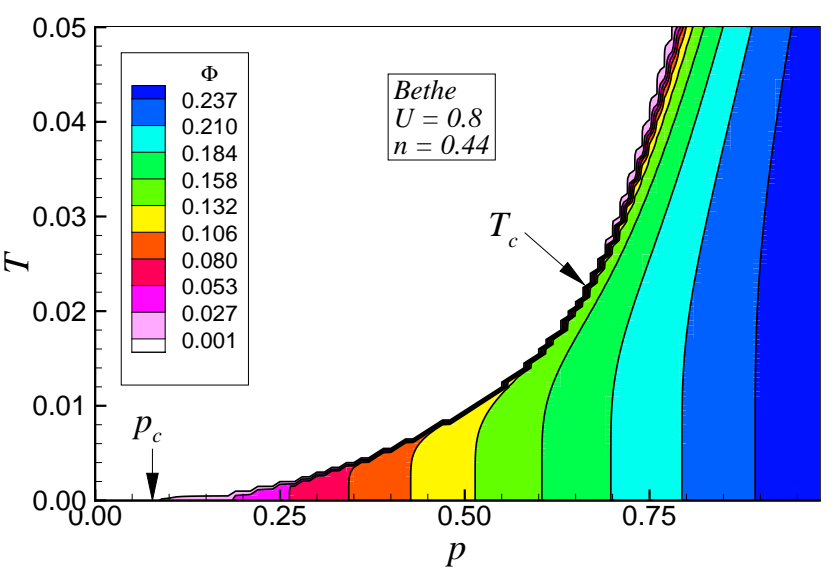

(b)

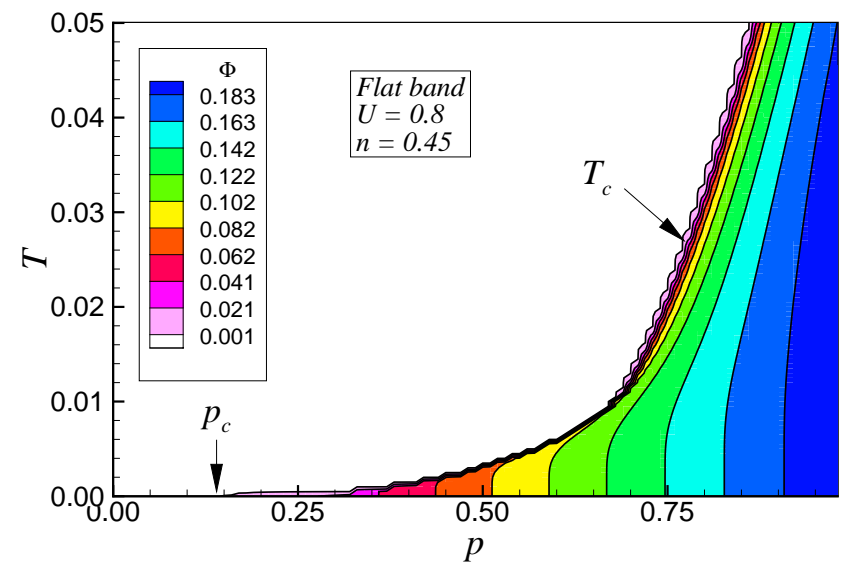

(d)

FIG. 4: (color online) Phase diagram in the $p-T$ plane. Plots show contours of constant pairing $\Phi$. The nearly straight line marked $T_{c}$ represents a continuous transition. The attractive interaction $U=0.8$ in all cases. (a) Bethe lattice with $n=0.072$ (b) Bethe lattice with $n=0.44$ (c) Flat band with $n=0.125$ (d) Flat band with $n=0.45$.

the panels in Fig. (4), the transition to a regime of vanishing pairing amplitude takes place by an abrupt (first order) transition (see, for example, region $0.2 \leq p \leq 0.6$ in Fig. 4(a)). On increase of the active fraction, a second regime appears (see, for example, region $0.6 \leq p \leq 0.75$ in Fig. (4) where there are two transitions. In this regime, with increase of temperature from zero, there is a first order transition where $\Phi$ (and $\Delta$ ) undergoes a sudden jump and obtains a smaller non zero value. With further increase of temperature the pairing amplitude vanishes continuously to zero. Interestingly, the first order line in the $p-T$ plane appears to end at a "critical point" (such as that marked by $C$ in Fig. प(a)). With further increase of the active fraction, a third regime is attained $(p>0.75$ in Fig. 4(a), for example), where $T_{c}$ depends essentially linearly on $p$, and the transition is continuous. These observations can be clearly seen by a study of Fig. 5 which shows a plot $\Delta(T) / \Delta(0)$ as a function of $T / T_{c}$, where the three types of behaviour are shown. Indeed, the features are generic and do not appear to depend on the shape of the bare density of states; they are clearly seen in both the Bethe lattice and flat band cases. The "sizes" of the three regimes are, however, strongly affected by the electron filling $n$. This is most clearly seen in the flat band case where the critical value of $p_{c}$ is insensitive to electron filling. However, the finite temperature behaviour strongly depends on electron filling - compare Fig. 4(c) and Fig. 4(d)), in the latter case the second regime of active fraction with two finite temperature transitions is strongly suppressed.

\section{DISCUSSION AND CONCLUSION}

This section contains a summary of the results obtained in this paper, and a discussion of the full phase diagram of the AHII model. The present calculation of the phase diagram is based on a single site dynamical mean field theory like approach. The calculation shows for a certain range of the strength of the interaction parameter $U$ which depends on the bare band structure and electron filling, there is a critical active fraction $p_{c}$ 


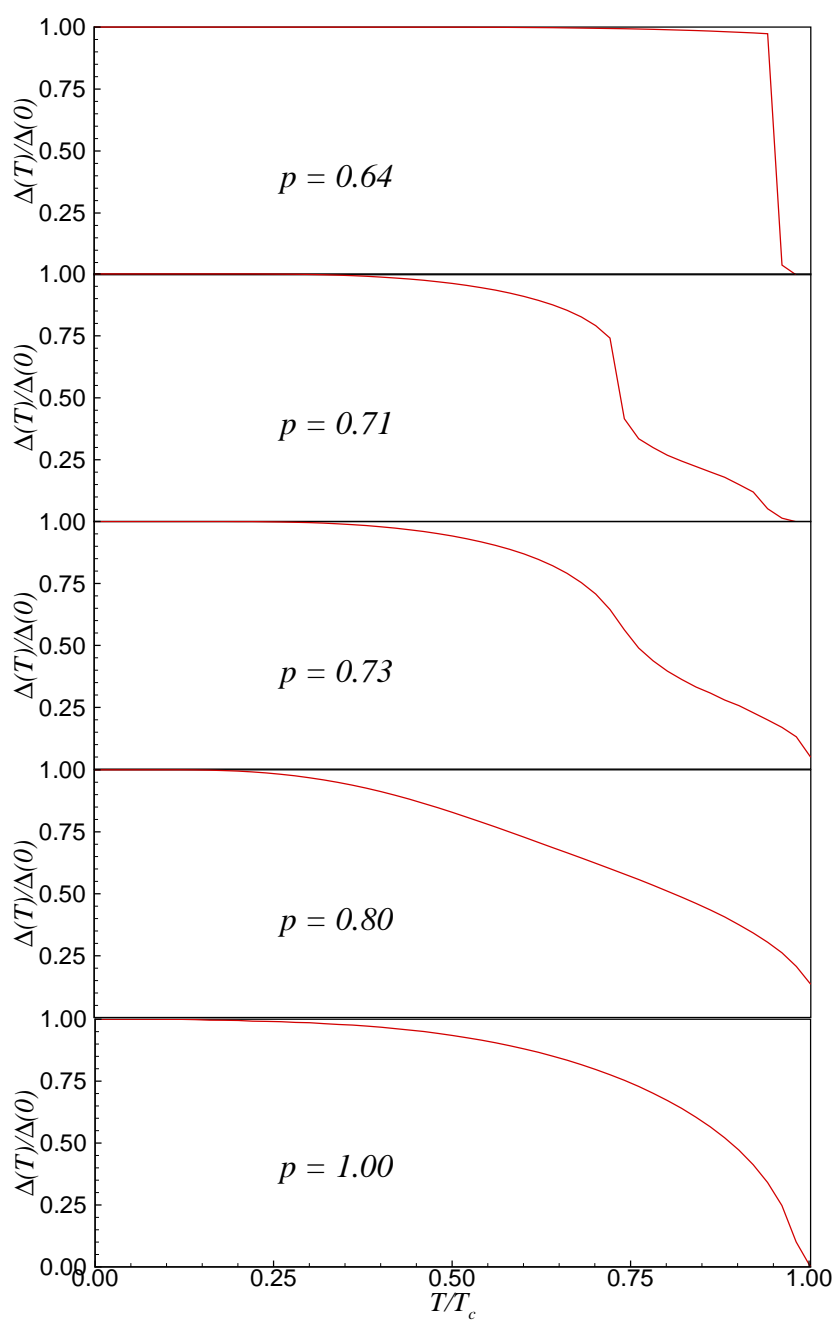

FIG. 5: (color online) Variation of $\Delta$ with temperature $T$ for the flat band $U=0.7, n=0.45$. The four panels show the behaviour of $\Delta$ for increasing values of the active fraction $p$. The critical value of the active fraction for this case $p_{c}=0.62$.

below which there is no pairing amplitude. However, for large enough values of the interaction parameter, even an infinitesimal value of the active fraction is sufficient to produce a non vanishing pairing amplitude. For active fraction $p$ greater than $p_{c}$ the electron spectral function shows a features with "mid-gap states", and the BCS singularity of the spectral function is smeared out.

For cases which show a nonvanishing pairing amplitude $\Phi$, three types of finite temperature behaviour are found. For $p$ close to $p_{c}$, there is a discontinuous transition at a finite temperature, and for $p$ close to unity, there is continuous transition (BCS like behaviour) to a state without pairing amplitude. There is a intermediate range of active fractions, where the transition to a non paired state takes place in two steps - "non-BCS behaviour". As the temperature is increased, there is a first order transition to a state with smaller $\Phi$. Further increase of temperature causes a continuous transition to a state

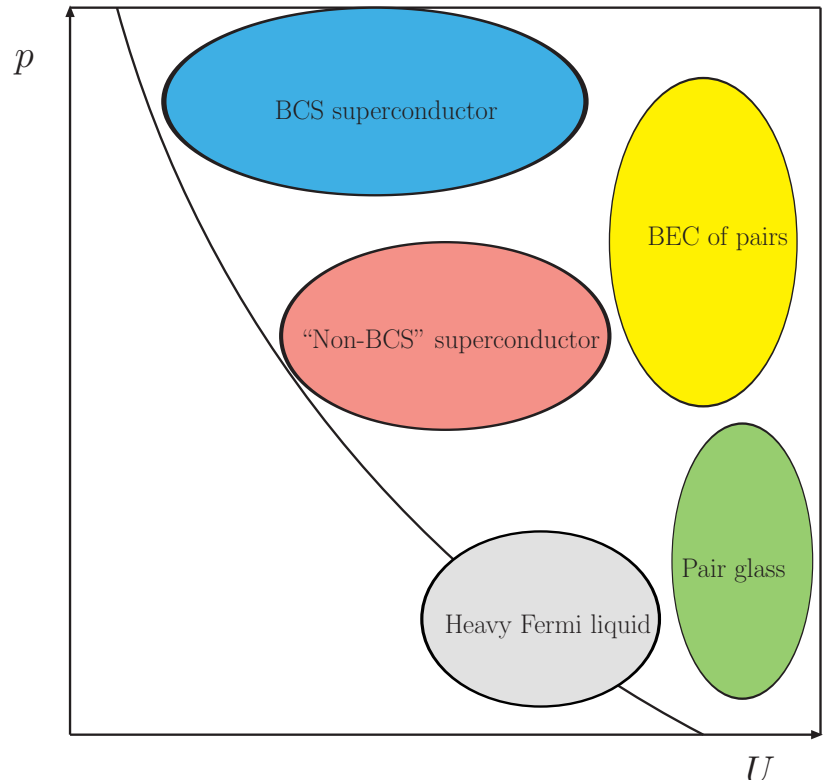

FIG. 6: (color online) Schematic phase diagram of AHII. The region indicated by "Non-BCS superconductor" indicates the unconventional superconducting behaviour found in the calculations presented in this paper. The region indicated by "Heavy Fermi Liquid" can be inferred from the work of Taraphder and Coleman $^{27}$. The region with "large $U$ " and large $p$ result in a "BEC of pairs" as is known from the work of Randeria ${ }^{45}$, and from the estimate of superfluid stiffness given in fig. 7. The region with large $U$ and small $p$ is speculated to have a "pair glass" ground state.

with no pairing amplitude. It is tempting to speculate that the state attained up on the first order transition has a pairing amplitude, but no superconductivity. The physical picture of such a state is that of "puddles of electrons" with non-zero pairing amplitude without a global phase necessary for superconductivity. Such a state is likely to show "psuedo-gap" like features, for example, a reduced spin susceptibility. Clearly, this finding of the present calculation needs more attention, and the region of the phase diagram where this phenomenon is found needs further detailed investigation. It is interesting to note that calculations based on BdGMF 35 also show a regime of $U$ and $p$ which show anomalous behaviour of $\Delta$ as a function of $T$.

The present formulation is based on a single site formulation and averages over all the spatial correlations. However, as noted above, a very interesting region in the phase diagram is revealed, and suggests possibility for further investigation. Further, the approximate treatment based on the saddle point approximation does not include quantum fluctuations. It is believed that the inclusion of these quantum fluctuation effects are not likely to change the qualitative features of the present single site calculation; this is suggested by the iterated perturbation theory based dynamical mean field theory of the attractive Hubbard model $\underline{\underline{46}}$ It must be noted that most 


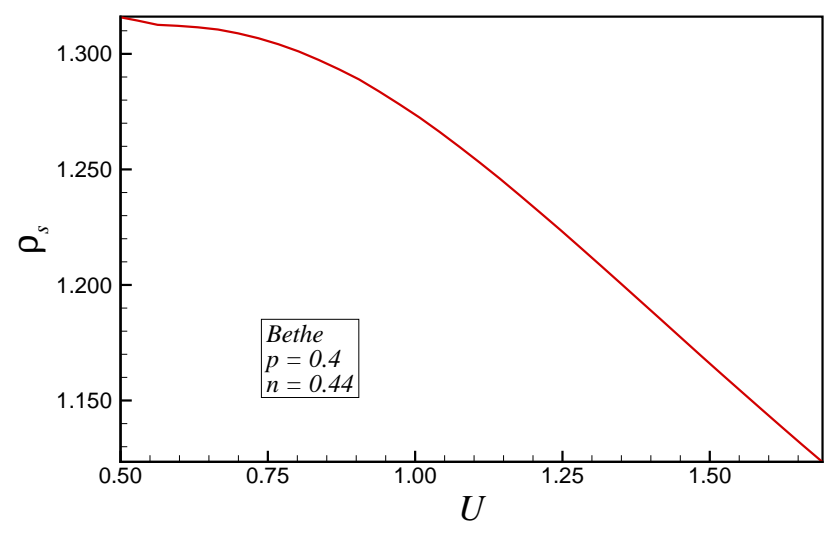

FIG. 7: (color online) Dependence of the superfluid density $\rho_{s}$ on the interaction strength $U$ in the Bethe lattice with $n=0.44$ and $p=0.4$.

of the previous work cited above are based on two dimensional systems, mostly square lattices. Long wavelength fluctuations, crucial in two dimensional systems, cannot be treated within the present framework. The present work, therefore, is more applicable to higher dimensional systems such as negative- $U$ centre systems etc.

The paper is concluded with a discussion of the complete phase diagram of the AHII model. Based on the calculations presented here and on published results quoted earlier, the nature of the electronic state in different regions of the parameter states can be inferred. In the regions with nonzero pairing amplitude (as obtained from the present calculation), different types of electronic states may be found as indicated in Fig. 6. For intermediate $U$ (compared to the bare bandwidth) and large $p$ a BCS superconductor is obtained. On the other hand for smaller value of the active faction $p$, a "non-
BCS" superconductor is obtained (as discussed above), with the possibility of a high temperature "pseudo-gap" phase. For larger values of the interaction strength there is a crossover from BCS to BEC like behaviour where the electrons form pairs and Bose condense ${ }^{45}$ In the present calculation, this behaviour is inferred by the calculation of an estimate (based on the kinetic energy) of the superfluid density $\stackrel{46}{\square}$ As shown in Fig. [7, the superfluid density $\rho_{s}$ falls with increasing $U$ indicating a crossover from BCS to BEC (Bose-Einstein condensation) behaviour. The effect of the random attractive interaction in this BEC regime needs a more careful investigation than that given here. For low values of the active fraction and intermediate values of the interaction strength, the most likely ground state is a heavy Fermi liquid 27 engendered by the charge Kondo effect. It is also possible that for small fillings, large interaction strengths, one could obtain a pair glass, where electrons are localized at the negative $U$ centers. Clearly, the "boundaries" of the regions indicated in the phase diagram will be determined by the third factor in the problem, namely electron filling. The nature of the electronic states in different parameter regimes of the AHII model does resemble various systems discussed in the introductory section. It will be interesting also to explore the possibility of a direct experimental realization of the AHII model in cold atom optical lattices.

\section{Acknowledgement}

The author thanks A. Ghosh, H. R. Krishnamurthy, T. V. Ramakrishanan and T. Senthil for discussions, and S. Bhowmick and S. Pathak for comments on the manuscript. Generous support for this work by DST, India through a Ramanujan grant is gratefully acknowledged.
* Electronic address: shenoy@physics.iisc.ernet.in

1 E. Sigmund and K. A. Müller, eds., Phase separation in cuprate superconductors (Springer, Heidelberg, 1994).

2 C. Howald, P. Fournier, and A. Kapitulnik, Physical Review B 64, 100504 (2001).

3 S. H. Pan, J. P. O'Neal, R. L. Badzey, C. Chamon, H. Ding, J. R. Engelbrecht, Z. Wang, H. Eisaki, S. Uchida, A. K. Gupta, K.-W. Ng, E. W. Hudson, K. M. Lang, and J. C. Davis, Nature 413, 282 (2001).

4 T. Hanaguri, C. Lupien, Y. Kohsaka, D.-H. Lee, M. Azuma, M. Takano, H. Takagi, and J. C. Davis, Nature 430, 1001 (2004).

5 M. Vershinin, S. Misra, S. Ono, Y. Abe, Y. Ando, and A. Yazdani, Science 305, 1995 (2004).

${ }^{6}$ K. McElroy, J. Lee, J. A. Slezak, D.-H. Lee, H. Eisaki, S. Uchida, and J. C. Davis, Science 309, 1048 (2005).

7 K. McElroy, D.-H. Lee, J. E. Hoffman, K. M. Lang, J. Lee, E. W. Hudson, H. Eisaki, S. Uchida, and J. C. Davis, Physical Review Letters 94, 107005 (2005).
${ }^{8}$ K. K. Gomes, A. N. Pasupathy, A. Pushp, S. Ono, Y. Ando, and A. Yazdani, Nature (2007).

${ }^{9}$ K. K. Gomes, A. N. Pasupathy, A. Pushp, S. Ono, Y. Ando, and A. Yazdani, Physica C 460-462, 212 (2007).

10 E. Dagotto, Nanoscale phase separation and colossal magnetoresistance (Springer-Verlag, Berlin, 2003).

11 N. D. Mathur and P. B. Littlewood, Physics Today pp. 25-30 (January 2003).

12 E. Dagotto, New Journal of Physics 7, 67 (2005).

13 V. B. Shenoy, D. D. Sarma, and C. N. R. Rao, Chem. Phys. Chem. 7, 2053 (2006).

14 V. B. Shenoy, T. Gupta, H. R. Krishnamurthy, and T. V. Ramakrishnan, Physical Review Letters 98, 097201 (2007).

15 V. B. Shenoy and C. N. R. Rao, Philosophical Transactions of the Royal Society A 366, 63 (2008).

16 E. Dagotto, Science 309, 257 (2005).

17 G. C. Milward, M. J. Calderón, and P. B. Littlewood, Nature 433, 607 (2005). 
18 P. W. Anderson, Physical Review Letters 34, 953 (1975).

19 N. T. Bagaraev, W. Gehlhoff, L. E. Klyachkin, A. M. Malyarenko, V. R. Romanov, and S. A. Rykov, Physica C 437-438, 21 (2006).

20 A. Taraphder, H. R. Krishnamurthy, R. Pandit, and T. V. Ramakrishnan, Physical Review B 52, 1368 (1995).

21 Y. Matsushita, H. Bluhm, T. H. Geballe, and I. R. Fisher, Physical Review Letters p. 157002 (2005).

${ }^{22}$ Y. Matsushita, P. A. Wianecki, A. T. Sommer, T. H. Geballe, and I. R. Fisher, Physical Review B 74, 134512 (2006).

23 M. Ma and P. A. Lee, Physical Review B 32, 5658 (1985).

24 A. Ghosal, M. Randeria, and N. Trivedi, Physical Review B 65, 014501 (2001).

25 D. Valdez-Balderas and D. Stroud, Physical Review B 74, 174506 (2006).

26 A. Garg, M. Randeria, and N. Trivedi, Nature Physics (2008), to be published.

27 A. Taraphder and P. Coleman, Physical Review Letters 66, 2814 (1991).

28 M. Dzero and J. Schmalian, Physical Review Letters 94, 157003 (2005).

29 T. M. Rice and L. Sneddon, Physical Review Letters 47, 689 (1981).

30 D. van der Marel and J. E. Mooij, Physical Review B 45, 9940 (1992).

31 G. Litak and B. L. Györffy, Physical Review B 62, 6629 (2000).

32 T. S. Nunner, B. M. Andersen, A. Melikyan, and P. J. Hirschfeld, Physical Review B 95, 177003 (2005).

33 B. M. Andersen, A. Melikyan, T. S. Nunner, and P. J. Hirschfeld, Physical Review B 74, 060501(R) (2006).

${ }^{34}$ K. Aryanpour, E. R. Dagotto, M. Mayr, T. Paiva, W. E. Pickett, and R. T. Scalettar, Physical Review B 73, 104518 (2006).

${ }^{35}$ K. Aryanpour, T. Paiva, W. E. Pickett, and R. T. Scalet- tar, Physical Review B 76, 184521 (2007).

36 B. Chatterjee and A. Taraphder, Pairing in disordered $s$ wave superconductors and the effect of their coupling, condmat/0702150.

37 D. Hurt, E. Odabashian, W. E. Pickett, R. T. Scalettar, F. Mondaini, T. Paiva, and R. R. dos Santos, Physical Review B 72, 144513 (pages 7) (2005), URL http://link.aps.org/abstract/PRB/v72/e144513

38 A. Georges, G. Kotliar, W. Krauth, and M. J. Rozenberg, Reviews of Modern Physics 68, 13 (1996).

39 V. Janiš and D. Vollhardt, Physical Review B 46, 15712 (1992).

40 J. Hubbard, Phys. Rev. Lett. 3, 77 (1959).

41 J. E. Hirsch and R. M. Fye, Physical Review Letters 56 , 2521 (1986).

42 R. J. Elliott, J. A. Krumhansl, and P. L. Leath, Reviews of Modern Physics 46, 465 (1974).

43 G. D. Mahan, Many-particle Physics (Kluwer Academic/Plenum Publishers, 2000).

44 M. Tinkham, Introduction to Superconductivity (McGrawHill, 1996).

${ }^{45}$ M. Randeria, in Bose Einstein Condensation, edited by A. Griffin, D. Snoke, and S. Stringari (Cambridge University Press, 1994).

46 A. Garg, H. R. Krishnamurthy, and M. Randeria, Physical Review B 72, 024517 (2005).

47 A. Georges, Condensed matter physics with light and atoms: Strongly correlated cold fermions in optical lattices (2007), cond-mat/0702122.

48 I. Bloch, J. Dalibard, and W. Zwerger, Many-body physics with ultracold gases, cond-mat/0704.3011.

${ }^{49} \mathrm{~W}$. Ketterle and M. W. Zwierlein, Making, probing and understanding ultracold fermi gases (2008), condmat/0801.2500. 